\title{
EL MERCOSUR COMO UNA PRIORIDAD DE LA POLÍTICA EXTERIOR BRASILEÑA
}

\author{
Miriam Gomes Saraiva*
}

El proceso de integración entre Brasil y Argentina, que tuvo como punto marco importante la firma del Tratado de Asunción para la formación del Mercosur (Mercado Común del Sur) en 1991, significó un punto de inflexión de la política exterior brasileña. La opción de un país que mantiene, tradicionalmente, un comportamiento externo bastante multilateralizado con vistas a colocarse como un actor global, por medio de un proceso de integración importante de carácter subregional, suscita de por sí una serie de preguntas. ¿Por qué una alternativa subregional? ¿Cuáles fueron los factores que, en un momento importante de transformación del orden internacional y de ajustes económicos internos en los Estados latinoamericanos, incidieron sobre esta opción? ¿Cuál es el papel de esta aproximación en los marcos de la política exterior brasileña?

Sin pretender dar cuenta en su totalidad de la complejidad de estas indagaciones, y trabajando desde una perspectiva brasileña, este artículo intenta identificar cómo se dio la evolución del proceso de integración entre Brasil y Argentina; cuáles fueron los factores de carácter tanto externo como interno que actuaron sobre el comportamiento brasileño en estos casos; cómo el Mercosur se coloca en la agenda de la política exterior brasileña; así como señala, de forma breve, algunos resultados de la firma del Tratado de Asunción.

\section{LA EVOLUCIÓN DEL PROCESO DE INTEGRACIÓN}

Las relaciones entre Brasil y Argentina, durante un siglo y medio desde sus procesos de independencia, fueron relaciones difíciles, entremezclándose períodos de rivalidad más clara con comportamientos -con poco éxito- de aproximación.

En 1941 hubo una tentativa de formación de un bloque austral a partir de la firma de un tratado comercial entre Brasil y Argentina, que no se hizo efectivo en función, sobre todo, de las posturas divergentes asumidas por ambos frente a la evolución de la Segunda Guerra Mundial. Entre 1951 y 1954, período de los gobiernos Vargas en Brasil y Perón en Argentina, tuvieron lugar intentos de articulación, que incluirían también a Chile, pero que no llegaron a concretarse. En 1961 los presidentes Quadros y Frondizi firmaron el Convenio de Amistad y Consulta, como mecanismo de cambio de informaciones con vistas a la

Traducción del portugués: Ana Inés López-Accotto.

(*) Profesora de la Universidad del Estado de Río de Janeiro. Becaria recién doctorada del Consejo Nacional de Desarrollo Científico y Tecnológico, Brasil. superación de las divergencias y a la cooperación entre ambos países. En 1969, como un nuevo intento, fue firmado el Tratado de Bacia do Prata, que buscaba formas de cooperación en los campos económicos y de seguridad. Pero ya aparecían los primeros conflictos entre los dos países sobre la utilización del potencial de los ríos de Bacia del Río Paraná.

Los años 70 estuvieron marcados por las rivalidades político-militares que se materializaron en enfrentamientos sobre la utilización de los recursos fluviales. Por fin, en 1979 fue firmado el Acuerdo Tripartito Corpus-Itaipú, que compatibilizó la construcción de dos hidroeléctricas y solucionó el contencioso sobre el tema.

La década de los 80, al contrario, se caracterizó como un período de armonía y aproximación, sobre todo a partir de 1985, cuando se inició el proceso real y formal de integración entre los dos países. En este caso, los pasos del procedimiento de aproximación respondieron también a transformaciones en la coyuntura internacional, acompañando la tendencia más general en el sentido de la regionalización.

En el campo comercial, en función de la política argentina de finales de los años 70 de apertura económica y desindustrialización, se consolidó una balanza asimétrica. En ese proceso, Argentina perdió su importancia para las importaciones brasileñas, al mismo tiempo que Brasil se afirmaba como principal abastecedor de manufacturas para el mercado argentino. Pero en ese momento los conflictos comerciales ya no envenenaban las relaciones; había ya una voluntad política de encontrar soluciones técnicas para cada caso. El propio problema de la crisis de la deuda externa y la necesidad de generar superávits agravó la cuestión además de desorganizar las economías de la región. En el campo político, hubo avances en el sentido de la percepción de la existencia de intereses comunes, que permaneció latente en el ejemplo de la Guerra de las Malvinas. En este episodio, aunque Brasil haya condenado la utilización de la fuerza, reconoció el derecho argentino sobre las islas. El fin del gobierno militar argentino y la elección del presidente Alfonsín, en 1983, no comprometieron las relaciones.

Pero fue a partir de 1985, con el establecimiento de un gobierno civil también en Brasil, cuando la idea de integración alcanzó una forma más clara. Desde mediados hasta el final de los años 80, diversos factores de carácter tanto externo como interno incidieron en el comportamiento brasileño en relación a Argentina, favoreciendo el impulso del proceso de integración entre ambos y la consecuente estructuración del Mercosur. En el 
campo exterior, los cambios que ocurrieran en el orden internacional desde el inicio de la década y que se acentuaron con la caída del muro de Berlín, contribuyeron al fortalecimiento de las perspectivas de integración. A nivel interno, los mecanismos de superación de la crisis económica vinculada al problema de la deuda externa, la necesidad de actualización del parque productivo, los proyectos de apertura económica y la consolidación del gobierno democrático actuaron, a su vez, como incentivo.

En el contexto externo, a partir de mediados de la década, tuvieron lugar innovaciones tecnológicas en las economías de los países centrales que contribuyeron a la estructuración de un nuevo paradigma técnico-productivo. Esto contribuyó, por su lado, a un nuevo ordenamiento del comercio internacional, de la división internacional del trabajo, de la competitividad de las economías, así como sustituyó los criterios de ventaja comparativa que estuvieron en vigor hasta entonces. Ello tuvo un impacto negativo en las economías de los países periféricos -en particular en los casos de Brasil y Argentina- aumentando tanto la distancia tecnológica entre el Norte y el Sur y la consecuente marginalización del último ${ }^{1}$. También las transformaciones que comenzaban a ocurrir en el escenario internacional a nivel polìtico, el nuevo impulso vivido por las experiencias de integración en curso y las afinidades en la propia percepción del contexto internacional por parte de los dos países contribuyeron a la aproximación.

En la política doméstica, la consolidación democrática favoreción el proceso de integración aproximando a los dos gobiernos puesto que serviría como elemento fortalecedor de la democracia internamente y como garante de las instituciones democráticas en los países de la región. Como telón de fondo, la democratización sirvió para disipar las desconfianzas y las ideas de disputa hegemónica que habían marcado las relaciones entre Brasil y Argentina durante mucho tiempo ${ }^{2}$.

En relación a la economía nacional, los problemas derivados de la crisis de la deuda externa -tanto en el nivel de las actividades productivas y fenómenos inflacionarios, como en una baja de la tasa de inversión- y la alternativa de planes de estabilización heterodoxos, reforzaron la tendencia a la integración. Al mismo tiempo, las transformaciones en el paradigma técnicoproductivo que marcaron a la economía internacional trajeron problemas en un sistema productivo basado en ventajas comparativas en vías de superación como recursos y mano de obra barata.

En 1985, se firmó la Declaración de Iguazú, que hacía referencia a la voluntad política de acelerar el proceso de integración con una ampliación del comercio, cooperación industrial y defensa del pluralismo democrático. Este fue el marco políticodiplomático del inicio del proceso de integración y señaló la superación total de una visión de cuño más geopolítico que había marcado las relaciones a lo largo del siglo.

A partir de ahí, el proceso de aproximación comenzó a caminar con pasos firmes. En 1986 se firmó el Acta de Integración, seguida de dos Protocolos de Integración referidos a sectores

(1) Sobre los cambios en el comercio internacional, ver ROSALES, Osvaldo. "Escenarios y tendencias en el comercio intemacional". En: Roberto Russell (comp.), La agenda internacional en los años 90 Buenos Aires, GEL/RIAL, 1990.

(2) BARBOSA, Rubens Antonio y CESAR, Luís Fernando Panelli. "A integraçao sub-regional, regional e hemisférica: o esforçó brasileiro". En: Gélson Fonseca Jr. e Sérgio Henrique Nabuco de Castro. Temas de política externa brasileira II, vol.I. Sao Paulo, Paz e Terra, 1994. específicos de las dos economías. Ya en este período, y en base a una actuación importante de la propia cancillería brasileña, la tendencia negativa del comercio bilateral se revirtió, volviendo éste a crecer. Pero no se concretó un impacto mayor de la integración debido a los problemas internos vividos por ambos en el área económica y de la concentración de esfuerzos para superarlos. También los impasses estructurales resultantes de la asimetría entre los sectores productivos de los dos países, la falta de coordinación de sus políticas macro-económicas y las oscilaciones cambiarias dificultaron la integración de los dos mercados $^{3}$. En el campo de la política exterior, fue un período de convergencias con una actuación en los grupos de Cargena y Apoyo, la decisión del establecimiento de la Zona de Paz y Cooperación del Atlántico Sur y la política nuclear autónoma.

En 1988, más en función de la voluntad política de las partes, se firmó el Tratado de Integración, Cooperación y Desarrollo, que preveía la formación de un área de libre comercio en diez años.

\section{LAS TRANSFORMACIONES EXTERNAS E INTERNAS DE LOS AÑOS 90 Y EL MERCOSUR.}

En el paso a los años 90, nuevos factores de carácter tanto interno como externo contribuyeron a un incremento del proceso de integración. En el escenario internacional, la desestructuración del socialismo en el Este europeo y los cambios en el sistema económico internacional abrieron espacio para la conformación de un nuevo orden e, internamente, los intentos de reordenamiento de la economía basada en un proceso de apertura al liberalismo incidieron en el comportamiento brasileño frente al tema.

En el campo político el nuevo orden en gestación asumió carácter homogéneo, apuntando a la necesidad de que todas las sociedades participaran de normas internas comunes identificadas con el pluralismo democrático como forma legítima de organización. En el campo económico el paradigma neoliberal pasó a ser tanto el marco de referencia de la economía internacional como el orientador de la reformulación y ejecución de políticas de ajustes y cambios estructurales interiores de los Estados. Los estímulos y presiones para la apertura económica, la menor intervención gubernamental en la economía, la desregulación y equilibrio fiscal pasaron a ser frecuentes. La idea de economía de mercado vino acompañada de una internacionalización creciente de los circuitos productivos y de una transnacionalización del movimiento de capitales e inversiones.

Este proceso de conformación de un nuevo orden no superó el problema de las desigualdades en el sistema internacional; paralelamente, los problemas relativos al desarrollo de los países del sur perdieron importancia en la agenda internacional. En relación a América Latina, este proceso significó un retorno al papel de países de poco poder de negociación, además de la reducción de su participación en la economía internacional.

Dentro de este contexto de globalización del nuevo orden, los procesos de regionalización o integración ganaron un papel destacado, siendo percibidos como positivos tanto por el Norte como por el Sur. Para los países centrales, las experiencias de integración podrían servir como un instrumento de diálogo y

(3) Para una visión de esta etapa del proceso de integración, ver CAMARGO, Sonia de. A integraçäo do Cone Sul. Rio de Janeiro, IRIPUC/RJ, 1993 (Textos IRI, nº 13). 
como un incentivo y control para el proceso de apertura económica y de ajuste estructural en las economías de los países periféricos, así como serían percibidas como factor de estabilidad del sistema internacional ${ }^{4}$. Para el Sur, las incertidumbres surgidas por la conformación de un nuevo orden internacional llevaron a los Estados a buscar mecanismos alternativos de inserción exterior.

También se nota una rápida proliferación de agrupamientos regionales o subregionales de carácter económico y refuerzo de los ya existentes. Estos agrupamientos, no obstante, asumen un carácter diferente de los procesos de integración vividos en los años 60 y 70. Se caracterizan por la apertura exterior con la liberalización de las economías también en relación a terceros países y, al mismo tiempo, tratan de volverse más atractivos para el capital externo en general. La opción por un proceso de integración con un carácter abierto y funcionando como canal de inserción en la economía internacional fue considerado como un mecanismo importante en el arco de las acciones externas.

Para América Latina específicamente, el agotamiento de los mecanismos preferenciales en el interio de la ALADI y la superación de políticas exteriores de cuño autonomista y orientadas por intereses económicos de corto plazo por parte de los países de la región, abrió espacio para los esquemas sub-regionales. En el cambio de década hubo una reiniciación del proceso de integración del Mercado Común Centroamericano y del Pacto Andino, junto con la formación del NAFTA, del Mercosur y de asociaciones más flexibles como el G-3 (México, Colombia y Venezuela) o acuerdos de libre comercio, así como propuestas más generales como la Iniciativa para las Américas, del presidente Bush. En el campo político, los esfuerzos de articulación se materializaron en la organización del Grupo de Río.

Internamente, la falta de un modelo de desarrollo económico basado en la sustitución de importaciones junto a los problemas financieros recurrentes de la crisis de la deuda externa llevaron a la redefinición del proyecto de desarrollo. La opción por un proceso de apertura de la economía con vistas a un crecimiento articulado con los insumos y mercados externos evidenció la necesidad de renovar el parque productivo como garantía de una inserción más competitiva en la economía internacional. Y la integración se situó como mecanismo de acceso a mercados externos y elemento capaz de impulsar en el sentido de las transformaciones y de la mayor eficiencia en el sistema productivo interno5.

El ascenso de los gobiernos de Collor y Menem aceleró el proceso de aproximación. Ambos -y el gobierno argentino con más éxito-, pasaron a implementar políticas de corte liberal orientadas a la apertura de las economías hacia el exterior, liberalización comercial, privatización y desregulación, contribuyendo a la adopción del modelo de integración con apertura hacia el exterior.

Otro elemento que ya se delineaba en este momento fue la participación de sectores empresariales en el proceso de integración. La propia crisis económica y los problemas inflacionarios experimentados por Brasil y la amenaza de marginalización de

(4) Para una visión de la Unión Europea sobre las experiencias de integración entre países del Sur BATALLER, Francisco. "Regional integration and trade". En: Commission of the European Communities, European economy; the European Community as a world trade partner. Bruxelas, 1993- aporta elementos interesantes.

(5) BARBOSA, Rubens Antonio. "Integraçao hemisférica: notas sobre os desafíos e as oportunidades para o Brasil”. En : Os novos blocos econômicos: desafios e oportunidades. Rio de Janeiro, Fundaçäo Getúlio Vargas, 1993. pp.137-146. la región del comercio internacional, flujos financieros e inversiones en escala, incentivaron a las élites económicas a buscar soluciones de desarrollo en el proceso de integración. ${ }^{6}$ En este caso el proyecto de integración podría disminuir los impactos y el propio ritmo de una apertura al exterior.? De esa forma, al contrario de las experiencias de la década de los años 60, cuando la conducción estaba concentrada en diplomáticos y muchas veces sin un respaldo económico en el ámbito doméstico, en el inicio de los años 90 los agentes económicos internos se mostraron interesados en una aproximación a Argentina -u otros socios eventuales.

En este contexto, en 1990 se firmaron el Acta de Buenos Aires -que limitaba el plazo de formación de un mercado común bilateral para el final de 1994- y el Acuerdo de Complementación Económica, que consolidaba la integración en el ámbito de la ALADI (Asociación Latinaoamericana de Integración). En seguida, Uruguay y Paraguay fueron incorporados a las negociaciones y, a principios de 1991, se firmó el Tratado de Asunción, con el proyecto de formar un mercado común en la fecha definida en el Acta de Buenos Aires, a través de un programa de liberalización comercial y libre circulación de bienes, servicios y factores productivos.

\section{EL MERCOSUR EN EL MARCO DE LA POLÍTICA EXTERIOR BRASILEÑA}

A partir de la firma del Tratado de Asunción, la profundización del proceso de integración con sus socios del sur pasó a ser un elemento fundamental para Brasil.

Desde la vertiente económica, en el campo interno con la economía brasileña en una fase de esfuerzos para estabilizarla, una unión aduanera (y más a largo plazo un mercado común) venía a favorecer la articulación de su proyecto de desarrollo contribuyendo a generar una economía de escala y más especializada, con mayores ventajas comparativas y eficiencia en la producción de mayor variedad de bienes. Al mismo tiempo, incentivaría una supuesta concurrencia mayor ${ }^{8}$.

Por otro lado, en lo que se refiere a su inserción externa, el Mercosur, basándose en un proyecto de integración abierta al exterior, podría contribuir en relación al comercio exterior y en cuanto polo de atracción de inversiones privadas exteriores. Para las inversiones externas, un mercado más amplio y con mayor estabilidad, en general, atrae capitales. ${ }^{9}$

El comercio entre los socios, en un primer momento, no apareció como elemento principal en función de la tendencia de Brasil de posicionarse como un global trader, con su comercio exterior multilateralizado y, por lo tanto, de la inviabilidad de

(6) Sobre esta cuestión, y en relación al Mercosur en términos más abarcativos, ver ALMEIDA.

(7) La postura de parte del empresariado nacional que resiste a un proceso de apertura es una cuestión importante para un artículo sobre las negociaciones relativas al proceso de integración con Argentina dentro del aparato de Estado brasileño y con los agentes económicos.

(8) Estas son, entre otras, ventajas posibles de una unión aduanera citadas en CHUDNOVSKY Daniel y PORTA, Fernando. "En torno a la integración económica argentino-brasileña". Revista de la CEPAL, $\mathrm{n}^{\circ} 39$. Santiago de Chile, dic. 1989.

(9) En 1989, las economías de Brasil, Argentina, Uruguay y Paraguay juntas representaban cerca del $50 \%$ del Producto Bruto, del producto industrial y del comercio intrazonal de América Latina. Y el 33\% del comercio exterior general de la región. PEÑA, Felix. "El mercosul y la integración en las Américas". Revista del Ministerio de Relaciones Exteriores y Culto, Año 1, $\mathrm{n}^{\circ} 1$. Buenos Aires, Instituto del Servicio Revista del Mimisterio de Re Exterior de la Nación, 1992. 
concentrarlo en una sola región ${ }^{10}$. Sin embargo, en la práctica, terminó funcionando con uno de los sectores donde la integración avanzó más.

En relación al intercambio económico con socios externos en términos más generales, uno de los canales de diálogo más importantes de política internacional ya en el cambio hacia los años 90, fue el de las negociaciones establecidas entre grupos de países. En este caso, el Mercosur desempeñaría un papel fundamental. Estos diálogos podrían ser llevados a cabo con otros esquemas de integración latinoamericanos o con otros grupos, como en el caso de la Unión Europea (con la que el diálogo fue establecido después de la conformación del Mercosur). Con esto se favorecería, entre otras cosas, la estrategia brasileña de contactos con mercados desarrollados, colocándose en mejores condiciones para enfrentarse a un mercado internacional competitivo y con barreras comerciales.

En términos políticos, el Mercosur podría funcionar también como un elemento de refuerzo de la capacidad brasileña de negociación, proporcionándole mayor peso en la arena internacional. La propia complejidad asumida por las relaciones internacionales con su diversidad de temas y actores, junto con la necesidad de estructurar una forma de inserción internacional más creativa, apuntaba la importancia de coordinar acciones con socios más próximos. En este momento la política externa brasileña buscaba la construcción de su legitimidad internacional, para lo cual el Mercosur podría ser un instrumento. En los marcos de un mercado integrado, Brasil estaría siendo coherente con las condiciones más generales de la globalización y de la formación de bloques regionales.

De este modo, se volvió importante para Brasil trabajar a partir de un acuerdo de integración, lo que le permitiría consolidar tanto la posición de negociación con terceros Estados como en un contexto de integración más amplio del continente latinoamericano. En lo que se refiere a los temas más políticos, la conformación de un acuerdo subregional podría favorecer la proyección externa de sus países miembros y, por lo tanto, de Brasil. La propia armonización de las relaciones de Brasil con Argentina (buscada desde principios de los años 80) ya se presentaba como un factor relevante en el estilo de inserción en el escenario internacional perseguido por la política exterior brasileña.

En relación a América Latina, en el campo político, el Mercosur podría proporcionar a la diplomacia brasileña un mejor posicionamiento en el espacio regional, mayor estabilidad y la consolidación de las instituciones democráticas estructuradas en los años 80. Significaba definitivamente la sustitución de la disputa por la hegemonía en el área a favor de una situación de cooperación e interdependencia regional. En el sector comercial, el Mercosur podría ser un elemento capaz de abrir camino para, más tarde, formar un área de libre comercio en América del Sur ${ }^{11}$.

En contraposición a los beneficios señalados, la conformación del Mercosur no debería significar una pérdida de soberanía. La consolidación del proceso de integración no debería obstaculizar los espacios de actuación externa en términos individuales de Brasil ya conquistados -negociaciones multilate-

(10) Sobre esta cuestión de Brasil como un global trader, ver BARBOSA, Rubens Antonio y CESAR, Luis Fernando Panelli." O Brasil como global trader". En : Gélson Fonseca Júnior y Sérgio HenCESAR, Luis Fernando Panelli." O Brasil c
rique Nabuco de Castro (org.), op. cit.

(11) El ALCSA fue un proyecto del gobierno de Itamar Franco que no tuvo continuidad. En el mismo período (1992), fue lanzada también la Iniciativa Amazónica. rales y actuación en foros internacionales- lo que reforzaba la idea de un Mercosur abierto al exterior y manteniendo espacios importantes a las soberanías nacionales. Igualmente, a nivel interno, la integración no podría subordinar las grandes decisiones económicas. Un proceso de integración con una estructura de carácter supranacional sería demasiado arriesgado, siendo preferible un proceso más flexible, gradual y orientado por una buena dosis de pragmatismo. En este momento, lo que sí aparecía como claro era la percepción de que Brasil tenía que ir al frente del proceso de integración aún sin tener definido del todo sus resultados a largo plazo; si se quedaba parado perdería el tren de la historia. ${ }^{12}$

\section{PRIMEROS RESULTADOS DESPUÉS DEL TRATADO DE ASUNCIÓN}

En relación con la evolución interna del Mercosur después de la firma del Tratado de Asunción, ésta se enfrentó con algunas limitaciones en función de las asimetrías existentes entre los socios en el campo económico y por el desequilibrio entre las políticas cambiarias de Brasil y Argentina. La coordinación de las políticas macroeconómicas, que era un elemento fundamental para la composición del mercado común, no fue considerada como prioritaria. La preservación de cierta autonomía en este campo tuvo un peso mayor. Por otro lado, el ritmo del proceso quedó limitado por tener lugar en un momento de crisis económica y planes de estabilización, subordinándose así a las "dictaduras de las coyunturas económicas"13.

Así, la opción fue por una integración pragmática y orientada hacia los sectores donde hubiese menos resistencia; en este caso, el sector comercial que, de hecho, avanzó bastante en los años que siguieron a la formación del Mercosur ${ }^{14}$.

De esta forma, a finales de 1994 los objetivos trazados en el Tratado no habían sido cumplidos y la fase de consolidación de la unión arancelaria o de transición, fue ampliada a más de diez años. Y con la aplicación del Plan de Estabilización en Brasil, las diferencias en relación a la apertura de la economía y al tipo de cambio se redujeron.

En términos institucionales, el Mercosur respondió íntegramente a las preocupaciones de la diplomacia brasileña en lo que se refiere al respeto del mantenimiento de la soberanía. Las instituciones estructuradas a partir del Tratado tenían un carácter estrictamente intergubernamental y fueron consolidadas en 1994 por un plazo mínimo hasta el final de la fase de transición.

En el campo de la política exterior, después de la firma del Tratado, las cancillerías de los países buscaron espacio de reconocimiento internacional presentando la nueva iniciativa a inter-

(12) El embajador José Vicente Pimentel, en una mesa redonda presentada en el Encuentro Anual de la Associaçäo Nacional de Pesquisa e Pós-Graduaçäo em Ciências Sociais, en octubre de 1995/Caxambú, la Associaçäo Nacional de Pesquisa e Pós-Graduaçäo em Ciências Sociais,
Brasil, hablaba de la "teoría de la bicicleta": quien para de pedalear, se cae.

(13) CORREA, Paulo Guilherme, MACHADO, Joäo Bosco M., VEIGA, Pedro da Motta. A agenda de Las Leñas e a integraço do Mercosul. Rio de Janeiro: FUNCEX, jul. 1992 (Texto para discusión $n^{\circ} 69$ ). La cuestión de las dictaduras de las coyunturas económicas fue planteada por Pedro Motta Veiga en una mesa redonda que tuvo lugar en la Fundación Getúlio Vargas, por el CPDOC, en septiembre de 1995.

(14) Sobre la evolución económica del proceso de integración, ver ALMEIDA, Paulo Roberto e at.,

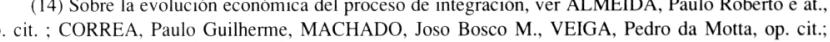
op. cit. ; CORREA, Paulo Guilherme, MACHADO, Joso Bosco M., VEIGA, Pedro da Motta, op. cit.,
VAZ, Alcides Costa. "Las alianzas regionales: Mercosur, Nafta y la Unión Latinoamericana". En: ArgenVAZ, Alcides Costa. "Las alianzas regionales: Mercosur, Nafta y la Unión Latinoamericana". En: Argen-
tina y Brasil en el Mercosur. Políticas comunes y alianzas regionales. Buenos Aires, Grupo Editor Latinoamericano, 1995 
locutores externos ${ }^{15}$. Esta estrategia presentó resultados más inmediatos en lo que se refiere a la actuación colectiva frente a algunos temas de agenda económica, donde hubo avances significativos a pesar de la postura más proteccionista mantenida por Brasil en su comercio exterior. La conformación de una Tarifa Externa Común llegó a abarcar, en 1995, el 85\% del comercio exterior de los países miembros; ello fue acompañado de una revisión en la trayectoria aperturista de Argentina ${ }^{16}$.

Las negociaciones de acuerdos comerciales con terceros Estados o grupos de Estados, por su parte, tuvieron un papel importante. Desde 1991 fue firmado un acuerdo con los EUA (conocido como cuatro + uno) en el marco de la Iniciativa para las Américas, y hubo gestiones diplomáticas con el Pacto Andino y con países asiáticos. Pero lo más importante fue el establecimiento de un diálogo con los países de la Unión Europea, institucionalizado como Acuerdo Marco Inter-Regional a finales de 1995.

En el campo de la política internacional en el mundo de la posguerra fría es donde las posiciones convergentes fueron más difíciles; sobre este espacio de articulación, el Tratado no hace estipulaciones concretas. Las divergencias se hicieron sentir en temas relevantes como las relaciones con Estados Unidos, el embargo norteamericano a Cuba, la ampliación de poderes de la Organización de Estados Americanos (OEA) y la composición del Consejo de Seguridad de las Naciones Unidas. El punto donde los frutos de una coordinación se hicieron presentes fue en la articulación desarrollada por Brasil y Argentina en el área de la política nuclear.

\section{CONCLUSIÓN}

La superación de las rivalidades que permeaban las relaciones de Brasil con Argentina y la formación del Mercosur fue un hecho nuevo y de mucha importancia para la política exterior brasileña, como la adopción de un nuevo paradigma en las relaciones exteriores de Brasil. En este caso, la profundización de la integración regional fue la cara externa de la transición democrática y del proceso de liberalización de la economía en la búsqueda de revigorizar el parque industrial brasileño de acuerdo con los nuevos parámetros internacionales. Frente a las transformaciones en el escenario internacional, Brasil buscó adaptarse a la evolución de esta nueva realidad.

En el campo de la política externa, con el ascenso del presidente Fernando Henrique Cardoso, el comportamiento de Brasil en la búsqueda de una mayor articulación con socios externos en el escenario internacional se presentó de forma más incisiva. Se notan esfuezos de la diplomacia brasileña en términos más políticos en el campo multilateral y con vistas a consolidarse en el campo comercial como un global trader. En este arco de acción, el Mercosur siguió ocupando una posición destacada, como una de las prioridades de la política exterior brasileña, tanto por lo que puede significar en términos de desarrollo nacional y de inserción brasileña en la economía internacional, como por su papel como instrumento de legitimación, fortalecimiento y canal de actuación externa de Brasil.

\section{RESUMEN}

En este artículo se presentan los antecedentes, constitución y primeros momentos del proceso de integración emprendido por Brasil, Argentina, Paraguay y Uruguay. Desde una perspectiva brasileña, la autora señala de qué manera una historia caracterizada por las tensiones diplomáticas fue convirtiéndose en un presente definido por metas comunes. También analiza los factores externos e internos que impulsaron a Brasil a la estrategia de la integración. Finalmente resume algunos de los avances económicos e institucionales conseguidos a lo largo del proceso y que definen el modelo de inserción de la economía brasileña en la mundial.

\section{ABSTRACT}

In this article there are displayed the antecedents, constitution and first moments of the process of integration undetaken by Brazil, Argentina, Paraguay and Uruguay. From a brazilean perspective, the author specifies the way in which a history of diplomacy tensions ends with common goals. There are also described the external and internal factors which impulsed Brazil into the strategy of integration. Finally, the author resumes some of the economic and institutional advances got during the process which define the model of insertion of brazilean economy in the world market. 